\title{
On the Maturity of Parallel Applications for Asymmetric Multi-Core Processors
}

\author{
Kallia Chronaki*1, Miquel Moreto*, Marc Casas*, Alejandro Rico ${ }^{\dagger}$, \\ Rosa M. Badia* $\ddagger$ Eduard Ayguadé*, Mateo Valero* \\ *Barcelona Supercomputing Center, \\ $\dagger A R M$, \\ ${ }^{\ddagger}$ Artificial Intelligence Research Institute (IIIA) - \\ Spanish National Research Council (CSIC)
}

\begin{abstract}
Asymmetric multi-cores (AMCs) are a successful architectural solution for both mobile devices and supercomputers. By maintaining two types of cores (fast and slow) AMCs are able to provide high performance under the facility power budget. This paper performs the first extensive evaluation of how portable are the current HPC applications for such supercomputing systems. Specifically we evaluate several execution models on an ARM big.LITTLE AMC using the PARSEC benchnark suite that includes representative highly parallel applications. We compare schedulers at the user, OS and runtime levels, using both static and dynamic options and multiple configurations, and assess the impact of these options on the well-known problem of balancing the load across AMCs. Our results demonstrate that scheduling is more effective when it takes place in the runtime system level as it improves the baseline by $23 \%$, while the heterogeneous-aware OS scheduling solution improves the baseline by $10 \%$.
\end{abstract}

Keywords: parallel programming, scheduling, runtime systems, asymmetric multi-cores, hpc

\footnotetext{
${ }^{1}$ Corresponding authors:

kallia.chronaki@bsc.es (Kallia Chronaki), miquel.moreto@bsc.es (Miquel Moreto), marc.casas@bsc.es (Marc Casas), alejandro.rico@arm.com (Alejandro Rico)
}

Preprint submitted to Journal of Parallel and Distributed Computing December 18, 2018

(C) 2019 Elsevier. This manuscript version is made available under the CC-BY-NC-ND 4.0 license http://creativecommons.org/licenses/by-nc-nd/4.0/ 


\section{Introduction}

The future of parallel computing is highly restricted by energy efficiency [1]. Energy efficiency has become the main challenge for future processor designs, motivating prolific research to face the power wall. Using heterogeneous processing elements is one of the approaches to increase energy efficiency $[2$, 3]. Asymmetric multi-core (AMC) systems is an interesting case of heterogeneous systems to utilize for energy efficiency. These systems maintain different types of cores that support the same instruction-set architecture. The different core types are designed to target different (performance or power) optimization points $[4,5,6]$.

AMCs have been mainly deployed for the mobile market. Mobile processors are also utilized in HPC platforms aiming to energy savings [7]. Asymmetric mobile SoCs combine low-power simple cores (little) with fast out-of-order cores (big) to achieve high performance while keeping power dissipation low. Another area where AMCs have been successful is the supercomputing market. The Sunway TaihuLight supercomputer topped the Top500 list in 2016 using AMCs. In this setup, big cores, that offer support for speculation to exploit Instruction-Level Parallelism (ILP), run the master tasks such as the OS and runtime system. Little cores are equipped with wide Single Instruction Multiple Data (SIMD) units and lean pipeline structures for energy efficient execution of compute-intensive code.

Like in other heterogeneous systems, load balancing and scheduling are fundamental challenges that must be addressed to effectively exploit all the resources in AMC platforms $[8,9,10,11,12,13]$. Mobile applications rely on multi-programmed workloads to balance the load in the system, while supercomputer applications rely on hand-tuned code to extract maximum performance. However, these approaches are not always suitable for generalpurpose parallel applications.

In this paper, we evaluate several execution models on an AMC using the PARSEC benchmark suite [14]. This suite includes parallel applications from multiple domains such as finance, computer vision, physics, image processing and video encoding. We quantify the performance loss of executing the applications as-is on all cores in the system. The main challenge on executing these applications on an AMC is to maintain load balance. These applications were originally developed on homogeneous platforms and typically operate by dividing the workload on even units. Executing these equal work units

on an asymmetric system is expected to suffer due to load imbalance. 
To overcome this matter, we consider two possible solutions at the OS and runtime levels to exploit AMCs effectively. The first solution delegates scheduling to the OS. We evaluate the built-in heterogeneity-aware OS scheduler currently used in existing mobile platforms that automatically assigns threads to different core types based on CPU utilization. The main drawback of this approach is the overhead introduced by the thread migration, thus resulting in limited performance no matter the potential of the underlying system.

The second solution is to transfer the responsibility to the runtime system so it dynamically schedules work to different core types based on work progress and core availability. We evaluate the impact of using an inherently loadbalanced execution model such that of task-based programming models. Recent examples $[15,16,17,18,19,20,21,22,23]$ include clauses to specify inter-task dependencies and remove most barriers which are the major source of load imbalance on AMCs. Another approach of scheduling in the runtime system is to change the existing statically-scheduled work-sharing constructs for the applications implemented in OpenMP to use dynamic scheduling.

This paper provides the first to our knowledge comprehensive evaluation of representative parallel applications on a real AMC platform: the OdroidXU3 development board with ARM big.LITTLE architecture. We analyze the effectiveness of the aforementioned scheduling solutions in terms of performance, power and energy. We show why parallel applications are not ready to run on AMCs and how OS and runtime schedulers can overcome these issues depending on the application characteristics. Further we point out in which aspects the built-in OS scheduler falls short to effectively utilize the AMC. Finally, we show how the runtime system approach overcomes these issues, and improves the OS and static threading approaches by $13 \%$ and $23 \%$ respectively.

The rest of this document is organized as follows: Section 2 describes the evaluated AMC processor, while Section 3 provides information on scheduling at the OS and runtime system levels. Section 4 describes the experimental framework. Section 5 shows the performance and energy results and associated insights.Finally, Section 6 discusses related work and Section 7 concludes this work.

\section{The ARM big.LITTLE Architecture}

The ARM big.LITTLE $[24,10]$ is a state-of-the-art AMC architecture that has been successfully deployed in the mobile market. ARM big.LITTLE 


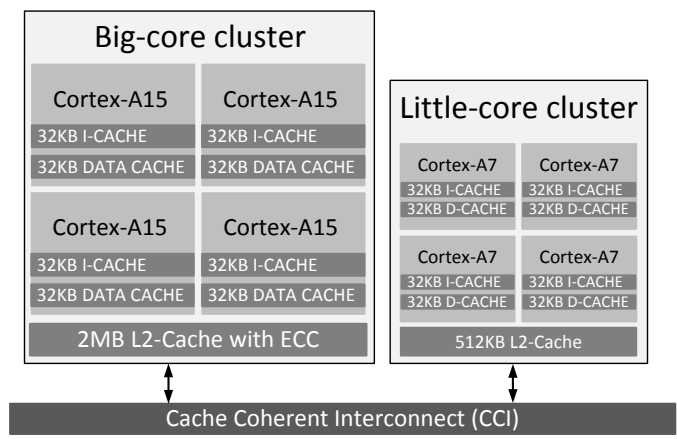

Figure 1: Samsung Exynos 5422 processor with ARM big.LITTLE architecture.

combines simple in-order cores (little) with aggressive out-of-order cores (big) in the same System-on-Chip (SoC) to provide high performance and low power. Big and little cores support the same instruction set architecture (ISA) so they can run the same binaries and therefore are easily combined within the same system. The little cores in a big.LITTLE system are designed targeting energy efficiency, while big cores are designed for high performance. Current cores implementing the ARMv7-A and ARMv8-A ISA support big.LITTLE configurations.

In this work, we use of one of the commercially available development boards featuring a big.LITTLE architecture: the Hardkernel Odroid-XU3 development board. As shown in Figure 1, the Odroid-XU3 includes an 8core Samsung Exynos 5422 chip with four ARM Cortex-A15 cores and four Cortex-A7 cores. In this SoC, there are two core clusters with caches that are shared among the cores of each cluster [25]. The four Cortex-A15 share a 2 MB 16-way 64-byte-cache-line L2 cache, while the Cortex-A7 cores share a 512 KB L2 cache. A single cache coherent memory controller (CCI) provides access to RAM to both clusters. The reason we use this platform instead of the more up-to-date Juno platform [26] is that even if the latter features the more advanced Cortex A53 [27] and Cortex A57 [28] cores, it is limited to six cores instead of the 8 cores in Odroid-XU3.

The Cortex-A7 cores in this SoC support dual-issue of instructions and their pipeline length is between 8 and 10 stages. The L1 instruction cache is $32 \mathrm{~KB}$ two-way set associative, with virtually indexed and physically tagged cache-lines that can hold up to 8 instructions. The L1 data cache is fourway set associative with physically-indexed and physically-tagged cache lines and uses a pseudo-random replacement policy [29]. Dynamic Voltage and Frequency Scaling (DVFS) techniques adjust the frequency of the little cores 
from $200 \mathrm{MHz}$ up to $1.4 \mathrm{GHz}$.

The Cortex-A15 cores in this SoC support triple-issue of instructions and their pipeline length is between 15 and 24 stages [30]. The L1 instruction and data caches of the Cortex-A15 are both $32 \mathrm{~KB}$ and 2-way set-associative with 64 byte cache lines [31]. DVFS techniques vary the frequency of the big cores from $200 \mathrm{MHz}$ up to $2 \mathrm{GHz}$. For the rest of the paper, we refer to Cortex-A15 cores as big and to Cortex-A7 cores as little.

\section{Scheduling in Asymmetric Multi-Cores}

Scheduling a set of processes on an AMC system is more challenging than the traditional process scheduling on SMCs. An efficient OS scheduler has to take into account the different characteristics of the cores and act accordingly [32]. There have been three mainstream OS schedulers for ARM big.LITTLE systems: cluster switching, in-kernel switch and global task scheduling, described in the next sections. In the case of parallel applications, dynamic scheduling at the runtime system level can be exploited to balance the workload among the different cores and is described in section 3.3. Finally, when all the scheduling mechanisms are disabled, the thread scheduling decisions are based on the static application-level scheduling.

\subsection{Cluster Switching and In-Kernel Switch}

In the Cluster Switching (CS) approach [24], only one of the clusters is active at any given time: either the cluster with little cores or the cluster with big cores executes. Thus, the OS scheduler operates on a de-facto symmetric multi-core with only four cores, namely the cores of the current active cluster. The policy to change the operating cluster is based on CPU utilization. When idle, background processes are executed on the little cores. When CPU utilization surpasses a threshold, all processes (foreground and background) are migrated to the big cluster. When running on the big cluster, if CPU utilization decreases below a given lower threshold, the entire workload is moved to the little cluster.

In the In-Kernel Switch (IKS) approach [33], each little core is paired with a big core and it is seen as a single core. On idle, background processes are run on little cores. When the CPU utilization on a given little core surpasses a threshold, the execution on that core is migrated to the big core. When the CPU utilization decreases on that big core below a given threshold, the execution migrates to the associated little core. Thus, at the same time, 
little and big cores may co-execute, but only one of each pair is active at a given point in time, effectively exploiting just half of the cores concurrently. For both CS and IKS, an enhanced cpufreq driver manages the switching within each core pair.

\subsection{Global Task Scheduling}

The Global Task Scheduling (GTS) [24] allows running applications on all cores in the asymmetric multi-core. In GTS, all cores are available and visible to the OS scheduler, and this scheduler is aware of the characteristics of the core types. Each process is assigned to a core type depending on its CPU utilization: high CPU utilization processes are scheduled to big cores and low CPU utilization processes to little cores. GTS also migrates processes between big and little cores when their CPU utilization changes. As a result, cores are active depending on the characteristics of the workload.

The key benefit of GTS is that it can use all the cores simultaneously, providing higher peak performance and more flexibility to manage the workload. In GTS tasks are directly migrated to cores without needing the intervention of the cpufreq daemon, reducing response time and minimizing the overhead of context switches. As a consequence, Samsung reported $20 \%$ improvement in performance over CS for mobile benchmarks [24]. Also, GTS supports clusters with different number of cores (e.g. with 2 big cores and 4 little cores), while IKS requires to have the same number of cores per cluster.

\subsection{Dynamic Scheduling in the Runtime}

Current programming models for shared memory systems such as OpenMP rely on a runtime system to manage the execution of the parallel application. In this work, we make use of two types of programming models: loop- and task-based. Loop-based scheduling distributes the iterations of a loop among the threads available in the system, following a traditional fork-join model. OpenMP supports loop-based scheduling through its parallel for directives. This clause implies a barrier synchronization at the end of the $\operatorname{loop}^{2}$, and supports either static or dynamic loop scheduling.

With static loop scheduling, the iterations of a loop are divided to as many chunks as the number of cores. Then, every core executes the assigned chunk, leading to a low-overhead static scheduling. In addition, OpenMP

\footnotetext{
${ }^{2}$ unless specified otherwise with the nowait clause
} 
supports dynamic loop scheduling. It generates more chunks than cores, and assigns them to the available cores at runtime. This is more suitable to asymmetric multi-core systems where the cores are not similar and a static iteration assignment would cause load imbalance.

Recent advances in programming models recover the use of task-based programming models to simplify parallel programming of multi-cores $[16$, $17,34,19,21]$. In these models the programmer splits the code in sequential pieces of work (tasks) and specifies the data dependencies among them. With this information the runtime system schedules tasks and manages synchronization. These models ease programmability [16, 17, 34, 19, 21, 22], and also increase performance by avoiding global synchronization points.

To evaluate this approach we make use of OpenMP tasking support [16]. OpenMP allows expressing tasks and data dependences between them using equivalent code annotations. It conceives the parallel execution as a task dependence graph (TDG), where nodes are sequential pieces of code (tasks) and the edges are control or data dependences between them. The runtime system builds this TDG at execution time and dynamically schedules tasks to the available cores. Tasks become ready as soon as their input dependencies are satisfied. The scheduling of the ready tasks is done in a first-comefirst-served manner, using a FIFO scheduler. Even though this scheduler is not aware of the task computational requirements or the core type and its performance and power characteristics, it can balance the load as long as there are ready tasks available thanks to the lack of global synchronization.

\subsection{Static Scheduling in the Application Level}

When the OS and runtime schedulers are disabled scheduling relies on the application. Current parallel applications generate software threads and rely on the operating system for the efficient mapping of these threads on the available cores. By disabling the operating system scheduler, each created thread is pinned on one of the cores and it is not allowed to migrate to another hardware component. Each pinned thread executes the work that the application is assigning to it statically. In this scenario, the application is responsible for the efficient parallelization, as it only depends on how the application is statically assigning the work on each of the software threads.

To evaluate this approach, we make use of applications that are implemented using the pthreads model. We then modify the code of the application so that each created thread is pinned to the next available processor. This way, 
Table 1: Benchmarks used from the PARSEC benchmark suite and their measured performance ratio between big and little cores

\begin{tabular}{|l|l|c|c|}
\hline Benchmark & \multicolumn{1}{|c|}{ Input } & Parallelization & Perf ratio \\
\hline \hline blackscholes & native & data-parallel & 2.18 \\
\hline bodytrack & native & pipeline & 4.16 \\
\hline canneal & native & unstructured & 1.73 \\
\hline dedup & $351 \mathrm{MB}$ data & pipeline & 2.67 \\
\hline facesim & native & data-parallel & 3.40 \\
\hline ferret & native & pipeline & 3.59 \\
\hline fluidanimate & native & data-parallel & 3.32 \\
\hline streamcluster & native & data-parallel & 3.48 \\
\hline swaptions & native & data-parallel & 2.78 \\
\hline
\end{tabular}

threads are statically assigned to processors and the operating system is not allowed to modify this.

\section{Experimental Methodology}

\subsection{Metrics}

All the experiments in this paper are performed on the Hardkernel Odroid XU3 described in Section 2. In our experiments we keep the frequencies of the cores static. This is in order to first, avoid machine overheating, and second to prevent the DVFS governor to dynamically modify the frequency of the cores during runtime, thing that would affect the reliability of the results. We make use of the cpufreq driver to set big cores at $1.6 \mathrm{GHz}$ and little cores at $800 \mathrm{MHz}$.

We evaluate seven configurations with different numbers of little (L) and big (B) cores, denoted L+B. For each configuration and benchmark, we report the average performance of five executions in the application parallel region. Then, we report the application speedup over its execution time on one little core. The variability of the results among the five runs is very small and the stdev of the speedup obtained ranges from 0 to 1.1 , averaging at 0.85 . Equation 1 shows the formula to compute this speedup.

$$
\operatorname{Speedup}(\mathrm{L}, \mathrm{B}, \text { method })=\frac{\text { Exec. time }(1,0, \text { method })}{\text { Exec. } \operatorname{time}(\mathrm{L}, \mathrm{B}, \text { method })}
$$

In this platform, there are four separated current sensors to measure, in real time, the power consumption of the A15 cluster, the A7 cluster, the GPU and DRAM. To gather power and energy measurements, a background daemon reads the machine power sensors periodically during the application 
execution with negligible overhead. Sensors are read at their refresh rate, every $270 \mathrm{~ms}$, and the values of A7 and A15 clusters' sensors are collected. With the help of timestamps, we correlate the power measurements with the application parallel region in a post-mortem process. The execution time overhead of the running daemon is measured and verified to be less than $3 \%$. 3 The reported power consumption is the average power tracked during five executions of each configuration, considering the application parallel region only. We then report average power in Watts along the execution.

Finally, in terms of energy and Energy Delay Product (EDP), we report the total energy and EDP of the benchmarks region of interest. To facilitate the explanation of these results and isolate the impact of the different system configurations on the energy consumption we normalize these results to the run on four little cores with static threading. Equations 2 and 3 show the formulas for these calculations.

$$
\begin{aligned}
\text { Normalized Energy }(\mathrm{L}, \mathrm{B}, \text { method }) & =\frac{\operatorname{Energy}(\mathrm{L}, \mathrm{B}, \text { method })}{\operatorname{Energy}(4,0, \text { static-threading })} \\
\text { Normalized } \operatorname{EDP}(\mathrm{L}, \mathrm{B}, \text { method }) & =\frac{\operatorname{EDP}(\mathrm{L}, \mathrm{B}, \text { method })}{\operatorname{EDP}(4,0, \text { static-threading })}
\end{aligned}
$$

\subsection{Applications}

With the prevalence of many-core processors and the increasing relevance of application domains that do not belong to the traditional HPC field, comes the need for programs representative of current and future parallel workloads. The PARSEC benchmark suite [14, 35] features state-of-theart, computationally intensive algorithms and very diverse workloads from different areas of computing. In our experiments, we make use of the original PARSEC codes together with a task-based implementation of nine benchmarks of the suite [36].

Table 1 shows the benchmarks included in the study along with their respective inputs, parallelization strategy and performance ratio between big and little cores per application. We are using native inputs, which are real input sets for native execution, except for dedup, as the entire native input file of $672 \mathrm{MB}$ and the intermediate data structures do not fit in the memory system of our platform. Instead, we reduce the size of the input file to 351 MB.

\footnotetext{
${ }^{3}$ In most cases this overhead is $1 \%$ with some applications reaching $2 \%$.
} 


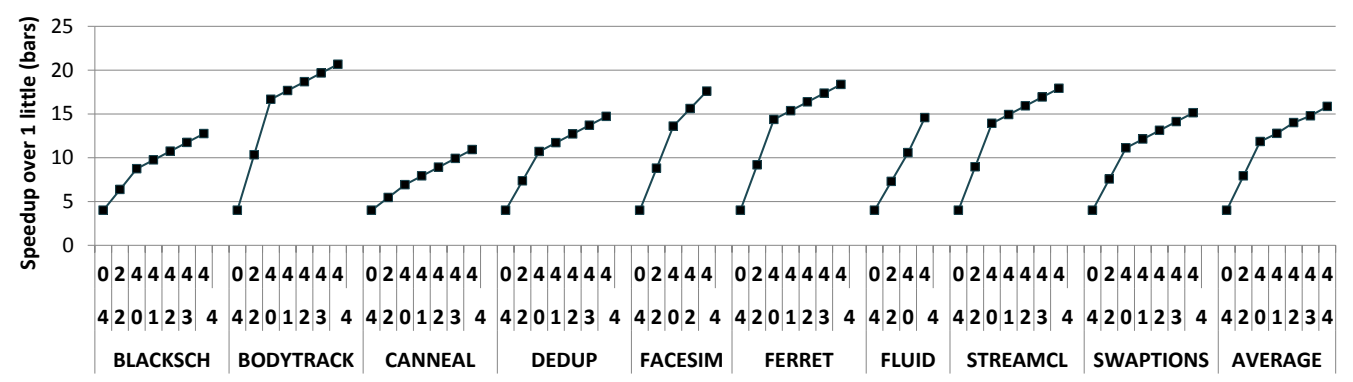

Figure 2: Ideal speedup over 1 little core according to Equation 4. Numbers at the bottom of $\mathrm{x}$ axis show the number of little cores, numbers above it show the number of big cores

The original codes make use of the pthreads parallelization model for all the selected benchmarks. The taskified applications follow the same parallelization strategy implemented with OpenMP 4.0 task annotations. The task-based implementation is done following two basic ideas: i) remove barriers where possible, by adding explicit data-dependencies; and ii) remove application-specific load balancing mechanisms, such as application-specific pools of threads implemented in pthreads and delegate this responsibility to the runtime.

When running on the big.LITTLE processor, each benchmark exhibits different performance ratios between big and little cores. These ratios tell us how many times faster a big core is compared to a little core. We measure the performance ratio of each application by executing it first on one big core and then on one little core, which corresponds to $\operatorname{Speedup}(0,1$, taskbased) in Equation 1. Table 1 also includes the observed performance ratio for each application. Bodytrack is the application that benefits the most from running on the big core with a performance ratio of $4.16 \times$. The out-oforder execution of the big core together with an increased number of in-flight instructions significantly improves the performance of this application. In contrast, canneal is the benchmark with the lowest performance ratio, $1.73 \times$, as this is a memory-intensive benchmark that does not benefit as much from the extra computation power of the big core. In general, performance ratios are above $2.5 \times$ for seven out of nine benchmarks, reaching $3.03 \times$ on average.

Taking into account these performance ratios, we can estimate the ideal speedup of the platform for each workload assuming a perfect parallelization strategy. This metric is useful for understanding the potential of each application irrespective of parallelization strategy and scheduling approach. It isolates 
the computations of each application and shows its ideal performance for each possible confuguration of the AMC. Equation 4 shows the equation for the ideal speedup over 1 little core computation according to the number of big (B) and little (L) cores.

$$
\text { Ideal speedup }(\text { workload, B, L) }=\text { B } \times \text { Perf_ratio }(\text { workload })+\mathrm{L}
$$

Figure 2 shows the ideal speedup of the system for each application for the varying numbers of cores. This speedup assumes that the applications are fully parallel with no barriers or other synchronization points. Thus, these speedups are an upper bound of the achievable application performance.

\section{Evaluation}

We measure execution time, power, energy and EDP of nine applications from the PARSEC benchmark suite [35]. We compare these metrics for three different scheduling approaches:

- Static threading: scheduling decisions are made at the application level. The OS is not allowed to migrate threads between the clusters of big and little cores.

- GTS ${ }^{4}$ : dynamic coarse-grained OS scheduling using the GTS scheduler integrated in the Linux kernel [24, 37] using the default PARSEC benchmarks.

- Task-based: dynamic fine-grained scheduling at the runtime level with the task-based implementations of the benchmarks provided in PARSECSs [36].

\subsection{Exploiting Parallelism in AMCs}

This section examines the opportunities and challenges that current AMCs offer to emerging parallel applications. With this objective, we first evaluate a system with a constant number of four cores, changing the level of asymmetry to evaluate the characteristics of each configuration. In these experiments, all applications run with the original parallelization strategy that relies on the user to balance the application (Static threading). We also evaluate the

\footnotetext{
${ }^{4}$ We choose to evaluate GTS instead of CS and IKS because it is the most advanced scheduling approach supported in the Linux kernel.
} 


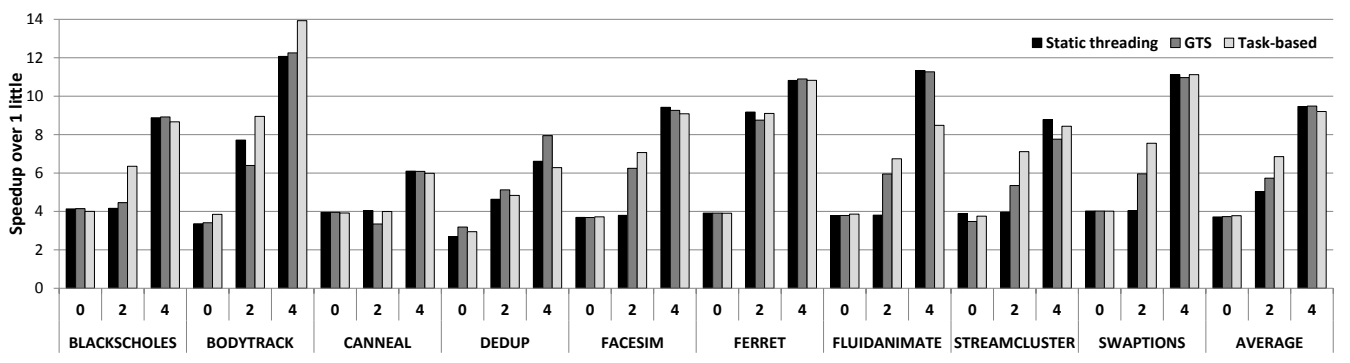

Figure 3: Execution time speedup over 1 little core for systems that consist of 4 cores in total with 0,2 and 4 big cores. Different schedulers at the application (static threading), OS (GTS) and runtime (task-based) levels are considered.

OS-based dynamic scheduling (GTS) and the task-based runtime dynamic scheduling (Task-based) for the same applications. The system configurations evaluated in this section are: i) Four little cores $(0+4)$; ii) Two big and two little cores (2+2); and iii) Four big cores (4+0)

For these configurations, Figure 3 shows the speedup of the PARSEC benchmarks with respect to running on a single little core. Figure 4 reports the average power dissipated on the evaluated platform. Finally, Figure 5 shows the total energy consumed per application for the same configurations. Energy results are normalized to the energy measured with four little cores (higher values imply higher energy consumptions). Average EDP results are also included in this figure.

Focusing on the average performance results, we notice that all approaches perform similarly for the homogeneous configurations. Specifically, applications obtain the best performance on the configuration $4+0$, with an average speedup of $9.5 \times$ over one little core. When using four little cores, an average speedup of $3.8 \times$ is reached for all approaches. This shows that all the approaches are effective for this core count. In the configuration 2+2, Static threading slightly improves performance (5.0× speedup), while GTS and Task-based reach significantly higher speedups: $5.9 \times$ and $6.8 \times$, respectively.

Contrarily, in terms of power and energy, the most efficient configuration is running with four little cores, as the performance ratio between the different cores is inversely proportional to the power ratio [10]. On average, all the approaches reach a power dissipation of $0.75 \mathrm{~W}$ for the $0+4$ configuration, while Task-based reaches $3.5 \mathrm{~W}$ for the $4+0$ configuration which is the one 


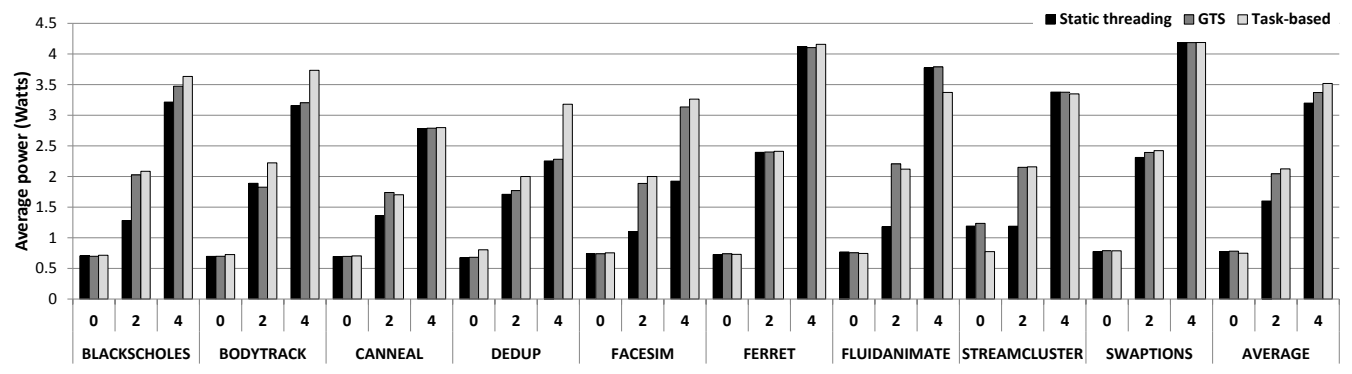

Figure 4: Average power measurements on a 4-core system with 0, 2, and 4 big cores.

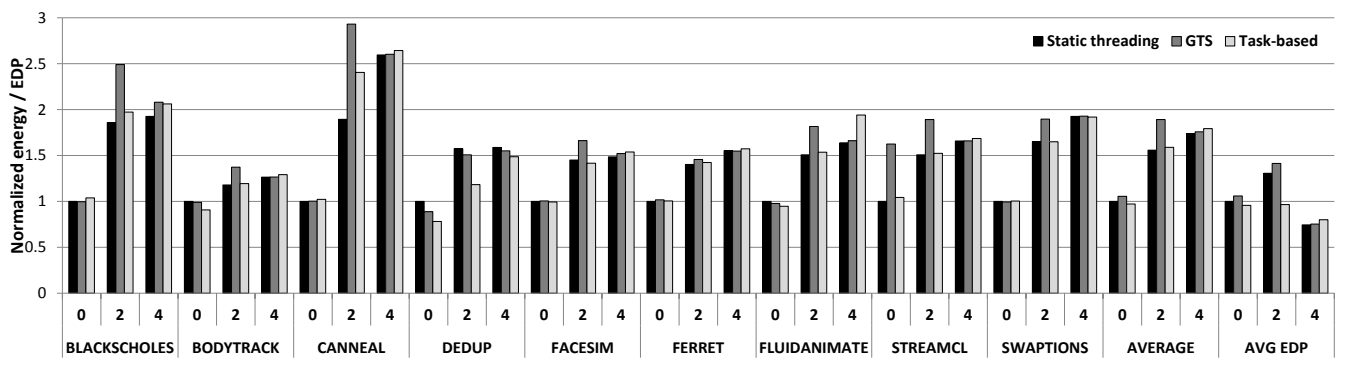

Figure 5: Normalized energy consumption and average EDP on a 4-core system with 0, 2, and 4 big cores. Static threading on 4 little cores is the baseline in both cases.

with the highest average power dissipation. In configuration $2+2$, average energy values for Static threading and Task-based are nearly the same, as the increase in power from $1.6 \mathrm{~W}$ to $2.1 \mathrm{~W}$ is compensated by a significant improvement in performance of $30 \%$.

Finally, in terms of EDP using the four big cores is the optimal, as the performance improvements compensate the increase in total energy. In configuration 2+2, Task-based achieves the same EDP results as in 0+4, but with $81 \%$ better performance. For the asymmetric configuration, Taskbased achieves the best performance-energy combination since its dynamic scheduling is effectively utilizing the little cores.

Next, we focus on the obtained results per benchmark. For applications with an extensive use of barriers (blackscholes, facesim, fluidanimate, streamcluster and swaptions) or with a memory intensive pattern (canneal), the extra computational power offered by the big cores in configuration $2+2$ is not exploited. As a result with Static threading performance is only slightly improved by $1 \%$ on average when moving from $0+4$ to the $2+2$ configuration. 
This slight improvement comes at the cost of much more power and energy consumption (79\% and $77 \%$ respectively). These results are explained threefold: i) load is distributed homogeneously among threads in some applications; ii) extensive usage of barriers force big cores to wait until little cores reach the barrier; and iii) high miss rates in the last-level cache cause frequent pipeline stalls and prevent to fully exploit the computational power of big cores. To alleviate these problems, the programmer should develop more advanced parallelization strategies that could benefit from AMCs, as performed in the remaining applications, or rely on dynamic scheduling at OS or runtime levels.

GTS is a suitable alternative for barrier-synchronized applications (blackscholes, facesim, fluidanimate, streamcluster and swaptions) when asymmetry is introduced. GTS enhances performance as it is dynamically migrating the threads around the cores depending on the CPU utilization. Thus it is expected that performance will increase compared to static threading for the asymmetric configuration. For these applications, the task-based approach further improves GTS for the asymmetric configuration. This is because task-based schedules tasks among threads which is much more efficient than scheduling threads among cores.

The three remaining applications are parallelized using a pipeline model (bodytrack, dedup, and ferret) with queues for the data-exchange between pipeline stages and application-specific dynamic load balancing mechanisms designed by the programmer. As a result, Static threading with these applications benefits from the extra computational power of the big cores in the configuration $2+2$. Since Static threading can already maintain load balance for these applications due to their implementation, there is no need for dynamic thread migration that is introduced by GTS. Using the task based approach, the code of the application is simplified allowing the application to express even more parallelism as the runtime system automatically allows the overlapping of the different pipeline stages. This can be verified by the fact that bodytrack obtains higher performance with the task based approach even for the symmetric configurations. On the asymmetric configuration, Task-based further improves the obtained performance, reaching a 13\% average improvement over GTS. Clearly, these applications benefit in performance by the increased number of big cores, while power and energy are increasing since the big cores are effectively utilized.

Generally, relying on the programmer to statically schedule asymmetric configurations does not report good results, as it is very hard to predict the system's behavior at application-level. Only applications that implement 
advanced features with user-level schedulers and load balancing techniques, can benefit from asymmetry, at the cost of programmability effort. Relying on the OS scheduler is a suitable alternative without code modifications, but relying on the runtime to dynamically schedule tasks on the asymmetric processor achieves much better performance, power and energy results.

\subsection{Adding Little Cores to an SMC}

In the following experiments, we explore if an application running on a symmetric multi-core (SMC) with big cores can benefit from adding small cores that help in its execution. Having more computational resources increases the ideal speedup a parallel application can reach, but it also introduces challenges at application, runtime and OS level. Thus, we examine how many small cores have to be added to the system to compensate the cons of having to deal with AMCs.

To evaluate this scenario, we explore configurations $4+0,4+1,4+2,4+3$ and $4+4$. In these experiments, the number of big cores remains constant (four), while the number of little cores increases from 0 to 4 . First we focus on the average results of speedup, power, energy and EDP, shown in Figure 6.

The speedup chart of Figure 6 shows that Static threading does not benefit from adding little cores to the system. In fact, this approach brings an average $6 \%$ slowdown when adding four little cores for execution $(4+4)$. This is a result of the static thread scheduling; because the same amount of work is assigned to each core, when the big cores finish the execution of their part, they become idle and under-utilized. GTS achieves a limited speedup of $8 \%$ with the addition of four little cores to the $4+0$ configuration. The addition of a single little core brings a $22 \%$ slowdown (from $4+0$ to $4+1)$ and requires three additional little cores to reach the performance of the symmetric configuration (4+3). Finally, the Task-based approach always benefits from the extra computational power as the runtime automatically deals with load imbalance. Performance improvements keep growing with the additional little cores, reaching an average improvement of $15 \%$ over the symmetric configuration when 4 extra cores are added.

The power chart of Figure 6 shows oppositional benefits among the three approaches. We can see that Static threading and GTS benefit from asymmetry, effectively reducing average power consumption. Static threading reduces power consumption when moving from the $4+0$ to the $4+4$ system by $23 \%$ while GTS does so by $6.2 \%$. On the other hand, the task-based approach 

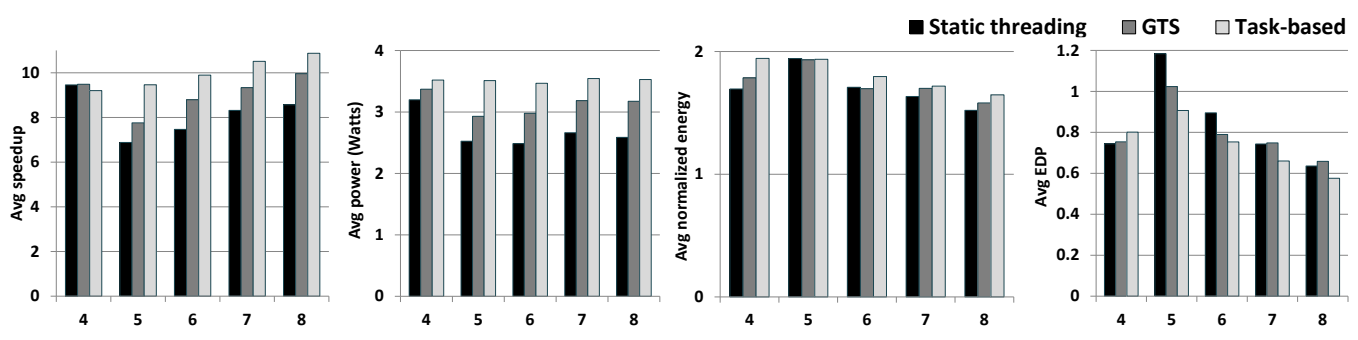

Figure 6: Average results when running on 4 to 8 cores with 4 of them big. Speedup is over 1 little core. Static threading on 4 little cores is the baseline of energy consumption and EDP

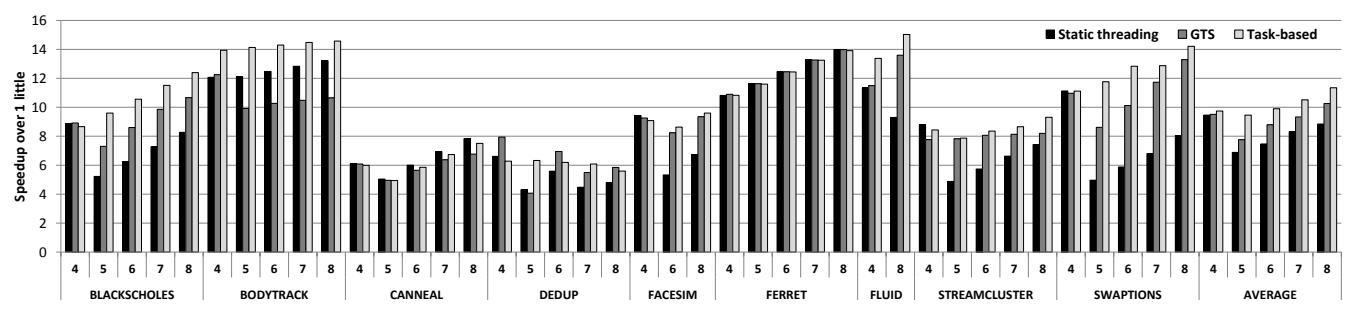

Figure 7: Speedup over 1 little core when running on 4 to 8 cores and 4 of them are big

keeps the big cores busy for most of the time so it maintains the average power nearly constant.

The reduction in power, results to reduced average energy in the case of Static threading in configuration 4+4, as shown on the energy chart of Figure 6. As discussed in Section 5.1, little cores are more energy efficient than big cores, at the cost of reduced performance. In all the approaches, at least two extra little cores are needed to reduce energy. In configuration $4+4$, energy is reduced by $14 \%$ for Static threading, $15 \%$ for GTS, and $16 \%$ for Task-based. Consequently, we can state that asymmetry reduces overall energy consumption.

To see the impact on both performance and energy efficiency we plot the average EDP on the rightmost chart of Figure 6. In this chart the lower values are the better. The task-based approach is the one that has the best performance-energy combination for the asymmetric configurations since it maintains the lowest EDP for all cases. Static threading manages to reduce the average EDP by $6 \%$ while GTS and task based approaches do so by $24 \%$ and $36 \%$ respectively.

Figure 7 shows a more detailed exploration of the performance results. As Table 1 shows, the applications with barrier synchronization are blackscholes, 


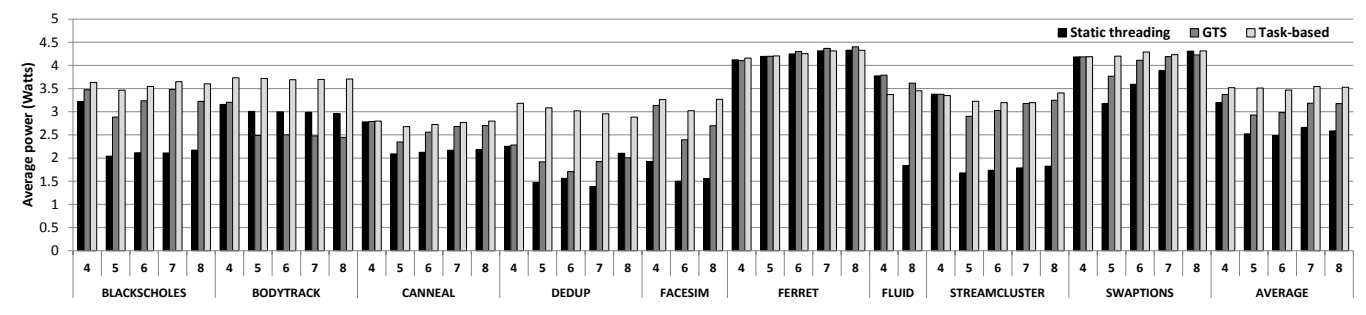

Figure 8: Average power when running on 4 to 8 cores and 4 of them are big

facesim, fluidanimate, streamcluster and swaptions. For these applications the most efficient system configuration with the Static threading approach is the 4+0. Little cores increase execution time due to load imbalance effects. GTS and task-based approaches overcome these issues by scheduling the load to the appropriate resources. The differences in the improvement of the task based and GTS solutions for these applications relies on the nature of each application and its parallel implementation. For example, swaptions, benefits more from the task based and GTS approaches than streacmluster. This is because the task graph of streamcluster presents multiple small parallel regions that are spawned and synchronized. Due to the multiple synchronization points, GTS and task-based cannot increase performance of streamcluster as much. Contrarily, swaptions has less synchronization points, thing that allows GTS and task-based to exploit asymmetry during its longer parallel regions.

Applications with more advanced load balancing techniques like pipelined parallelism (bodytrack, dedup and ferret), benefit of the asymmetric hardware and balance the load among all the cores. As a result, the performance of Static threading approach does not degrade when adding little cores as in the previous set of applications. In the case of bodytrack, GTS reduces performance by $15 \%$ when adding four little cores. We attribute this to the cost of the thread migration from one core to the other in contrast to the Static threading approach that does not add such overheads. Task based approach also avoids these overheads and improves the performance of bodytrack by efficiently scheduling the tasks among threads.

In the case of dedup, results show more variability. This benchmark is very $\mathrm{I} / \mathrm{O}$ intensive and, depending on the type of core that executes these I/O operations, performance drastically changes. In order to deal with this problem, a smarter dynamic scheduling mechanism would be required. 
Finally, canneal does not scale according to its ideal speedup reported on Figure 2 as it has a memory intensive pattern that limits performance.

Figure 8 shows the average power. The barrier-synchronized applications (blackscholes, facesim, fluidanimate, streamcluster and swaptions) reduce power because of their imbalance; since big cores have long idle times with the Static threading approach, they do not dissipate the same power as GTS and Task-based. For pipeline-parallel applications, both bodytrack and ferret maintain nearly the same power levels among the configurations for each scheduling approach. Dedup is an exception, as the results highly depend on the core that executes the aforementioned I/O operations. Yet, the effect of the lower power for Static threading is observed in all the benchmarks and is because the big cores are under-utilized.

\section{Discussion}

Sections 5.1 and 5.2 explored the potential of different scheduling approaches when used on various workloads on an AMC. It was proven that current applications are not ready to utilize an AMC and that adding little cores to an SMC with big cores presents significant challenges for the application, OS and runtime developers. Little cores increase load imbalance and can degrade performance as a result.

A dynamic OS scheduler such as GTS helps in mitigating load imbalance, providing an average performance increase of 10\%. Barrier synchronized applications benefit more from the GTS approach as the applications with more sophisticated scheduling techniques can utilize the little cores more efficiently even with Static threading. Task based approach offers the optimal performance results for all types of workloads. It improves Static threading by $20 \%$ on average by effectively balancing the load among big and little cores.

In terms of power and energy, the AMC provides significant benefits, although the SMC with little cores remains the most energy-efficient configuration. This is attributed to the differences of the designs of the big and little cores; little cores have been optimized for power efficiency while the design of the big cores targets higher performance levels at the cost of higher energy consumption. The answer to the question of which system configuration provides the best power-performance balance, can be found on the average EDP chart of Figures 5 and 6 , and is the use of the entire 8-core system with the Task based approach. 


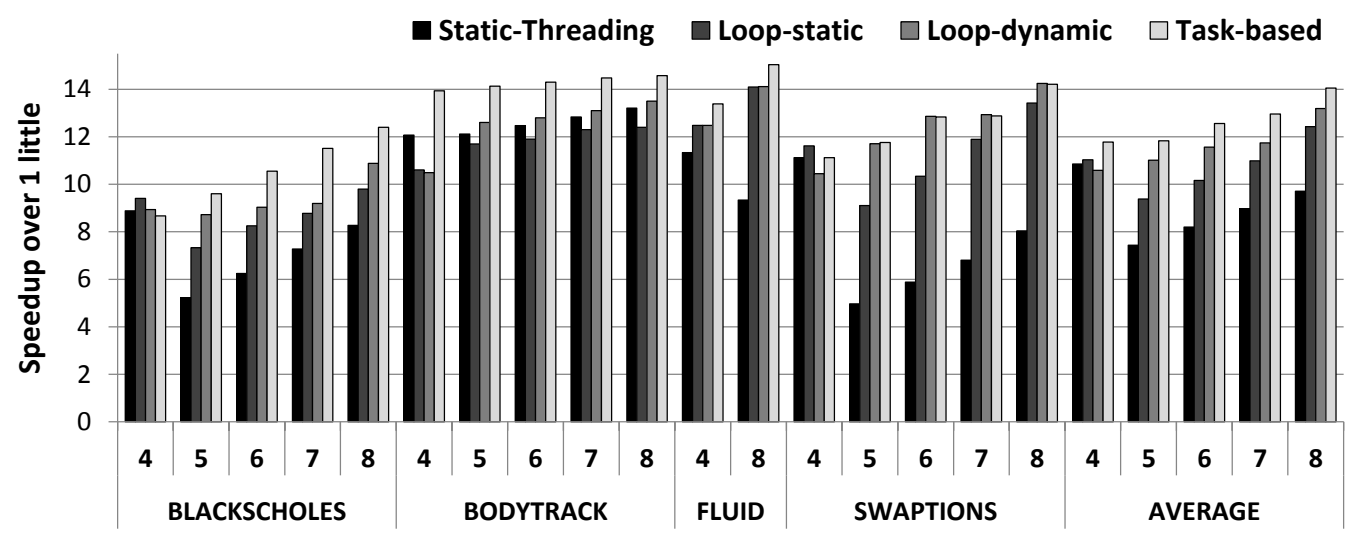

Figure 9: Speedup over 1 little core when running on 4 to 8 cores and 4 of them are big. Four different programming models are considered: Static threading using pthreads, parallel loops with static scheduling (loop static), parallel loops with dynamic scheduling (loop dynamic), and a task-based solution with dynamic scheduling (task-based).

\subsection{Programming Models for AMCs}

As we saw in the previous section, current implementations of parallel applications are not ready to fully take advantage of an AMC system. Applications that are statically threaded using the low-level pthreads library usually suffer from load imbalance since their implementations assume that the work has to be equally distributed among the available cores. Implementing advanced load balancing schemes, such as work pools, in pthreads requires a significant development effort.

As an alternative, many parallel applications are implemented using loopbased scheduling with the OpenMP parallel for directives. In this case, the runtime library is in charge of scheduling work to the available threads in the system, either statically or dynamically, as described in Section 3.3.

We compare these solutions to the task-based approach evaluated in the previous sections. Figure 9 shows the results obtained from running blackscholes, bodytrack, fluidanimate and swaptions on all the scheduling models: static threading, static loop scheduling, dynamic loop scheduling and task-based scheduling. We chose these applications as they are the only ones implemented using the OpenMP loop directives.

Looking at the average results in Figure 9, we can observe that the taskbased solution achieves the best results when the system is asymmetric. Taskbased improves the static threading by up to $59 \%$ on 5 cores, while dynamic 
loop scheduling improves by up to $54 \%$. The OpenMP version with static scheduling reaches an average $26 \%$ improvement over the static-threading approach with pthreads. The main reason for this improvement is that the OpenMP programming model by default allows the OS scheduler to migrate threads to cores. Thus, in this case, GTS is allowed to move threads from little to big cores or vice versa, which differs to the static threading that pins the threads to the cores. Similarly, loop-dynamic allows dynamic iteration scheduling as well as thread scheduling by GTS.

Taking a closer look to the results we observe that for bodytrack, an application with sophisticated parallelization techniques, static-threading achieves better results than loop-static. This is because the static-threading implementation contains specific parallelization techniques that cannot be completely expressed using the loop-static method. The loop-dynamic method improves performance for bodytrack by up to $4 \%$ due to the runtime decisions of the iteration execution, but the optimal solution is offered by the task-based approach that achieves up to $16 \%$ improvement over static-threading, due to the flexibility in expressing irregular parallelization strategies.

Blackscholes, fluidanimate and swaptions, consist of independent tasks and are a good fit for loop parallelism. The first observation is that all three applications benefit from the loop-static approach on an SMC with 4 big cores. Moreover, the task-based approach is still the optimal for blackscholes and fluidanimate, reaching up to $83 \%$ improvement over static threading for 5 cores, while for swaptions both task-based and loop-dynamic are efficient, improving the baseline by up to $2.3 \times$. Finally, fluidanimate, that is also a fine-grained application that consists of 128500 tasks, also benefits from the task-based approach. For this benchmark, static and dynamic loop scheduling achieve similar performance; this is due to the limited parallelism per parallel region, as the loop-based implementation consists of multiple barriers between small parallel regions, fact that diminishes the effect of dynamic vs static scheduling.

\section{Related Work}

There has been a lot of studies on AMC systems. Some works focus on the system design, while other works explore the challenges that appear in efficiently utilizing such a heterogeneous system. Kumar et al [38] present the idea of an AMC system and proposed a feedback-based way to dynamically migrate processes among the different cores. To determine the core that 
most effectively executed a workload, Kumar et al [4] proposed the use of sampling. This method minimizes the execution time of each single thread and increases performance. Other studies focused on the pipeline design of such AMCs and the area that should be devoted to each component in the system $[5,39]$. Other works on AMCs focus on hardware support for critical section detection [8] or bottleneck detection $[11,12]$. These approaches are orthogonal to the ones evaluated in this paper and could benefit from them to further improve the final performance of the system.

Process scheduling on AMCs is one of the most challenging topics in this area of study. Bias scheduling [40] is an OS scheduler that characterizes the running threads according to their memory or execution intensity. It then schedules the computation intensive threads to the big cores of the system while the memory intensive threads to the little cores of the system. The experimental evaluation is done on Intel Xeon processors and the heterogeneous system is emulated by changing the configuration of three out of the four cores of the processor. Cong et al propose the Energy-Efficient [41] OS scheduler based on energy estimation. The evaluation is performed on the Intel QuickIA [42] platform that integrates an Intel Xeon with an Atom processor. Van Craeynest et al. [43] propose the fairness-aware OS scheduler that focuses on AMC architectures. The performance impact estimation (PIE) scheduler [44] is based on the impact of MLP and ILP on the overall CPI and focuses on improving performance. The scheduler predicts the impact of each different core-type of the system on the MLP, ILP and it assumes hardware support for CPI. Rodrigues et al [45] propose a thread scheduling technique that estimates power and performance when deciding to assign a thread to a specific core of the heterogeneous system. Finally, Energy-Aware Scheduling (EAS) is an on-going effort in the Linux community to introduce the energy factor in the OS scheduler $[46,47]$. It is based on performance and power profiling to set performance and power capacities and let the Linux completely fair scheduler assign slots to processes considering the different core capacities. EAS is not yet part of the Linux kernel and, therefore, GTS is the most sophisticated state of the art scheduling method in production on current big.LITTLE processors.

Similar to OS scheduling approaches there have been many task scheduling approaches that are directed for utilizing AMCs. The Levelized Min Time [48] heuristic first clusters the tasks that can execute in parallel (levels) and then it assigns priorities to them, according to their execution time. The Dynamic Level Scheduling algorithm [49] assigns the tasks to the processors according 
to their dynamic level (DL). Heterogeneous Economical Duplication (HED) [50] duplicates the tasks in order to be executed on more than one cores but it then removes the redundant duplicates if they do not affect the makespan. CATS scheduler [51] is designed for AMCs like big.LITTLE and dynamically schedules the critical tasks to the big cores of the system to increase performance. Topcuoglu et al proposed the Heterogeneous Earliest Finish Time (HEFT) scheduler that statically assigns each task to the processor that will finish it at the earliest possible time. To do so, it keeps records with the task costs for each processor type. They also proposed the Critical Path on a Processor (CPOP) algorithm [52] that maintains a list of tasks and statically identifies and schedules the tasks belonging to the critical path to the processor that minimizes the sum of their execution times. The Longest Dynamic Critical Path (LDCP) algorithm [53] identifies the tasks that belong to the critical path and schedules them with higher priority.

All these works reflect the remarkable research that is taking place on AMCs. However we consider that their experimental evaluation is limited for three main reasons: i) The evaluation is done through a simulator or emulation of an AMC [38, 39, 5, 40, 43, 44, 45, 49, 48, 50, 8, 11, 12]; ii) The evaluated applications are either random task dependency graph generators or scientific kernels and micro-benchmarks $[49,52,53]$. iii) Their evaluation does not focus on power and energy consumption [4, 43, 44, 48, 51].

This paper includes a unique evaluation of performance, power and energy on a real AMC of real parallel applications. This paper also reflects the impact of using different big and little core counts which is not present in previous works [41].

\section{Conclusions}

This paper presented the first to tour knowledge extensive evaluation of highly parallel applications on an ARM big.LITTLE AMC system. The goal of this study was to identify whether such applications are ready to efficiently utilize an AMC system as well as finding the most appropriate software level for performing scheduling in order to maintain the load balance of the system. Quantifying such results requires a big effort in characterizing all parts of the evaluation including the applications as well as the scheduling approaches. The main findings of our work are the following:

- Current implementations of parallel applications using pthreads are not ready to to fully utilize an AMC. Our analysis covered 
a broad set of applications with different characteristics. From these, applications with highly sophisticated parallelization strategies such as parallel pipelines were able to exploit AMCs at the application level. However, this requires a significant programming effort and is not applicable to all workloads. The rest of the workloads are data-parallel applications that when moved to an AMC introduce load imbalance that limits their performance.

- A highly sophisticated asymmetry-aware OS scheduler is not the ideal solution to schedule parallel applications on AMCs. Our results demonstrated that GTS can only slightly improve performance of data-parallel applications. For applications with sophisticated parallelization strategies GTS fails to increase performance of the application as it introduces high overheads due to thread migration.

- Even if it is asymmetry-unaware, the task-based solution is the most appropriate as it allows dynamic load balancing and eliminates the thread migration costs. We saw that with a dynamic scheduling approach on the runtime system we have multiple benefits. First, it improves performance for all types of applications. In addition, there are cases where due to the increased programming flexibility in expressing parallelism, performance is improved (bodytrack). We further compared task-based solution against loop scheduling approaches on the runtime system and highlighted the benefits of the task-based solution.

As future work we aim to explore how the performance ratio between the types of cores affects the performance of the evaluated scheduling approaches. It is expected that increasing the performance ratio, the task-based approach is going to achieve even better results, as the load would be balanced again by dynamically assigning more work to the big cores. In this study, the performance-energy trade-off is something that needs to be taken into account as well.

\section{Acknowledgements}

This work has been supported by the RoMoL ERC Advanced Grant (GA 321253), by the European HiPEAC Network of Excellence, by the Spanish Ministry of Science and Innovation (contracts TIN2015-65316-P), 
by the Generalitat de Catalunya (contracts 2014-SGR-1051 and 2014-SGR1272), and by the European Union's Horizon 2020 research and innovation programme under grant agreement No 671697 and No. 779877. M. Moretó has been partially supported by the Ministry of Economy and Competitiveness under Ramon y Cajal fellowship number RYC-2016-21104. 


\section{References}

[1] P. Kogge, K. Bergman, S. Borkar, D. Campbell, W. Carson, W. Dally, M. Denneau, P. Franzon, W. Harrod, K. Hill, Others, Exascale Computing Study: Technology Challenges in Achieving Exascale Systems, Tech. rep., University of Notre Dame, CSE Dept. (2008).

[2] A. Lukefahr, S. Padmanabha, R. Das, F. M. Sleiman, R. Dreslinski, T. F. Wenisch, S. Mahlke, Composite cores: Pushing heterogeneity into a core, in: MICRO, 2012, pp. 317-328.

[3] Y. Wu, C. Gillan, U. Minhas, S. Barbhuiya, A. Novakovic, K. Tovletoglou, G. Tzenakis, H. Vandierendonck, G. Karakonstantis, D. Nikolopoulos, Heterogeneous servers based on programmable cores and dataflow engines, in: EnESCE, 2017.

[4] R. Kumar, D. M. Tullsen, P. Ranganathan, N. P. Jouppi, K. I. Farkas, Single-isa heterogeneous multi-core architectures for multithreaded workload performance, in: ISCA, 2004, pp. 64-75.

[5] S. Balakrishnan, R. Rajwar, M. Upton, K. K. Lai, The impact of performance asymmetry in emerging multicore architectures, in: ISCA, 2005, pp. 506-517.

[6] H. Wong, A. Bracy, E. Schuchman, T. M. Aamodt, J. D. Collins, P. H. Wang, G. Chinya, A. K. Groen, H. Jiang, H. Wang, Pangaea: A tightlycoupled ia32 heterogeneous chip multiprocessor, in: PACT, 2008.

[7] M. A. Laurenzano, A. Tiwari, A. Cauble-Chantrenne, A. Jundt, W. A. Ward, R. Campbell, L. Carrington, Characterization and bottleneck analysis of a 64-bit armv8 platform, in: ISPASS'16, 2016, pp. 36-45.

[8] M. A. Suleman, O. Mutlu, M. K. Qureshi, Y. N. Patt, Accelerating critical section execution with asymmetric multi-core architectures, in: ASPLOS, 2009, pp. 253-264.

[9] A. Fedorova, J. C. Saez, D. Shelepov, M. Prieto, Maximizing Power Efficiency with Asymmetric Multicore Systems, Communications of the ACM 52 (12).

[10] P. Greenhalgh, big.LITTLE Processing with ARM Cortex-A15 \& Cortex-A7, ARM White Paper. 
[11] J. A. Joao, M. A. Suleman, O. Mutlu, Y. N. Patt, Bottleneck identification and scheduling in multithreaded applications, in: ASPLOS, 2012.

[12] J. A. Joao, M. A. Suleman, O. Mutlu, Y. N. Patt, Utility-based acceleration of multithreaded applications on asymmetric CMPs, in: ISCA, 2013, pp. 154-165.

[13] N. Rajovic, P. M. Carpenter, I. Gelado, N. Puzovic, A. Ramirez, M. Valero, Supercomputing with Commodity CPUs: Are Mobile SoCs Ready for HPC?, in: SC, 2013.

[14] X. Zhan, Y. Bao, C. Bienia, K. Li, Parsec3.0: A multicore benchmark suite with network stacks and splash-2x, SIGARCH Comput. Archit. News 44 (5) (2017) 1-16.

[15] E. Ayguadé, N. Copty, A. Duran, J. Hoeflinger, Y. Lin, F. Massaioli, X. Teruel, P. Unnikrishnan, G. Zhang, The design of OpenMP tasks, IEEE TPDS 20 (3) (2009) 404-418.

[16] OpenMP architecture review board: Application program interface (2013).

[17] A. Duran, E. Ayguadé, R. M. Badia, J. Labarta, L. Martinell, X. Martorell, J. Planas, Ompss: a Proposal for Programming Heterogeneous Multi-Core Architectures., Parallel Processing Letters 21.

[18] B. Ren, S. Krishnamoorthy, K. Agrawal, M. Kulkarni, Exploiting vector and multicore parallelism for recursive, data- and task-parallel programs, in: PPoPP '17, New York, NY, USA, 2017, pp. 117-130.

[19] M. Bauer, S. Treichler, E. Slaughter, A. Aiken, Legion: Expressing locality and independence with logical regions, in: SC, 2012.

[20] K. Dichev, H. Jordan, K. Tovletoglou, T. Heller, D. Nikolopoulos, G. Karakonstantis, C. Gillan, Dependency-aware rollback and checkpoint-restart for distributed task-based runtimes, 2017.

[21] H. Vandierendonck, G. Tzenakis, D. S. Nikolopoulos, A unified scheduler for recursive and task dataflow parallelism, in: PACT, 2011. 
[22] H. Vandierendonck, K. Chronaki, D. S. Nikolopoulos, Deterministic scale-free pipeline parallelism with hyperqueues, in: SC, 2013.

[23] X. Tang, A. Pattnaik, H. Jiang, O. Kayiran, A. Jog, S. Pai, M. Ibrahim, M. T. Kandemir, C. R. Das, Controlled kernel launch for dynamic parallelism in gpus, in: HPCA'17, 2017, pp. 649-660.

[24] H. Chung, M. Kang, H.-D. Cho, Heterogeneous Multi-Processing Solution of Exynos 5 Octa with ARM big.LITTLE Technology, Tech. rep., Samsung Electronics Co., Ltd. (2013).

[25] L. Gwennap, Cortex-A75 Has DynamIQ Debut, Microprocessor Report (2017).

[26] ARM, Juno ARM Development Platform.

[27] K. Krewell, Cortex-A53 is ARM's next little thing (2012).

URL www.mpronline.com

[28] J. Bolaria, Cortex-A57 extends ARM's reach (2012).

URL www.mpronline.com

[29] ARM, Cortex-A7 MPCore, revision: r0p3 (2011).

URL http://infocenter.arm.com/help/topic/com.arm.doc. ddi0464d/DDI0464D_cortex_a7_mpcore_r0p3_trm.pdf

[30] J. Turley, Cortex-A15 eagle flies the coop (2011).

URL www.mpronline.com

[31] ARM, Cortex-A15 technical reference manual, revision: r2p0 (2011). URL http://infocenter.arm.com/help/topic/com.arm.doc. ddi0438c/DDI0438C_cortex_a15_r2p0_trm.pdf

[32] T. Cao, W. Huang, Y. He, M. Kondo, Cooling-aware job scheduling and node allocation for overprovisioned hpc systems, in: IPDPS'17, 2017, pp. $728-737$.

[33] Mathieu Poirier, In Kernel Switcher: A solution to support ARM's new big.LITTLE technology, Embedded Linux Conference 2013 (2013). 
[34] S. Zuckerman, J. Suetterlein, R. Knauerhase, G. R. Gao, Using a "Codelet" program execution model for exascale machines: Position paper, in: EXADAPT, 2011.

[35] C. Bienia, Benchmarking modern multiprocessors, Ph.D. thesis, Princeton University (2011).

[36] D. Chasapis, M. Casas, M. Moreto, R. Vidal, E. Ayguade, J. Labarta, M. Valero, PARSECSs: Evaluating the Impact of Task Parallelism in the PARSEC Benchmark Suite, TACO.

[37] B. Jeff, big.LITTLE Technology Moves Towards Fully Heterogeneous Global Task Scheduling, ARM White Paper.

[38] R. Kumar, K. I. Farkas, N. P. Jouppi, P. Ranganathan, D. M. Tullsen, Single-isa heterogeneous multi-core architectures: The potential for processor power reduction, in: MICRO, 2003, pp. 81-92.

[39] T. Y. Morad, U. C. Weiser, A. Kolodny, M. Valero, E. Ayguade, Performance, power efficiency and scalability of asymmetric cluster chip multiprocessors, IEEE Comput. Archit. Lett. 5 (1) (2006) 4-17.

[40] D. Koufaty, D. Reddy, S. Hahn, Bias scheduling in heterogeneous multicore architectures, in: EuroSys, 2010, pp. 125-138.

[41] J. Cong, B. Yuan, Energy-efficient scheduling on heterogeneous multicore architectures, in: ISLPED, 2012, pp. 345-350.

[42] N. Chitlur, G. Srinivasa, S. Hahn, P. Gupta, D. Reddy, D. Koufaty, P. Brett, A. Prabhakaran, L. Zhao, N. Ijih, S. Subhaschandra, S. Grover, X. Jiang, R. Iyer, Quickia: Exploring heterogeneous architectures on real prototypes, in: HPCA, 2012, pp. 1-8.

[43] K. Van Craeynest, S. Akram, W. Heirman, A. Jaleel, L. Eeckhout, Fairness-aware Scheduling on single-ISA Heterogeneous Multi-cores, in: PACT, 2013.

[44] K. Van Craeynest, A. Jaleel, L. Eeckhout, P. Narvaez, J. Emer, Scheduling heterogeneous multi-cores through performance impact estimation (pie), in: ISCA, 2012, pp. 213-224. 
[45] R. Rodrigues, A. Annamalai, I. Koren, S. Kundu, Scalable thread scheduling in asymmetric multicores for power efficiency, in: SBACPAD, 2012, pp. 59-66.

[46] M. Anderson, Scheduler Options in big.LITTLE Android Platforms.

[47] Ian Rickards and Amit Kucheria, Energy Aware Scheduling (EAS) progress update, https://www . linaro .org/blog/core-dump/energyaware-scheduling-eas-progress-update (2015).

[48] M. A. Iverson, F. Özgüner, G. J. Follen, Parallelizing Existing Applications in a Distributed Heterogeneous Environment, in: HCW, 1995.

[49] G. Sih, E. Lee, A Compile-Time Scheduling Heuristic for Interconnection-Constrained Heterogeneous Processor Architectures, IEEE TPDS 4 (2).

[50] A. Agarwal, P. Kumar, Economical Duplication Based Task Scheduling for Heterogeneous and Homogeneous Computing Systems, in: IACC, 2009 .

[51] K. Chronaki, A. Rico, R. M. Badia, E. Ayguadé, J. Labarta, M. Valero, Criticality-aware dynamic task scheduling for heterogeneous architectures, in: ICS, 2015, pp. 329-338.

[52] H. Topcuoglu, S. Hariri, M.-Y. Wu, Performance-Effective and LowComplexity Task Scheduling for Heterogeneous Computing, IEEE TPDS $13(3)$.

[53] M. Daoud, N. Kharma, Efficient Compile-Time Task Scheduling for Heterogeneous Distributed Computing Systems, in: ICPADS, 2006. 